\title{
Risk of obstructive sleep apnea and excessive daytime sleepiness in hospitalized psychiatric patients
}

This article was published in the following Dove Press journal:

Neuropsychiatric Disease and Treatment

26 April 2017

Number of times this article has been viewed

\author{
Farid R Talih' \\ Jean J Ajaltouni' \\ Hani M Tamim² \\ Firas H Kobeissy ${ }^{3}$ \\ 'Department of Psychiatry, \\ ${ }^{2}$ Department of Internal Medicine, \\ American University of Beirut Medical \\ Center, Beirut, Lebanon; ${ }^{3}$ Department \\ of Biochemistry and Molecular \\ Genetics, Faculty of Medicine, \\ American University of Beirut, \\ Beirut, Lebanon
}

Objectives: This study evaluated the risk of developing obstructive sleep apnea (OSA) and excessive daytime sleepiness (EDS) in hospitalized psychiatric patients at the American University of Beirut Medical Center (AUB-MC). Factors associated with OSA and EDS occurrence in this sample were also examined.

Methods: The Berlin questionnaire and the Epworth sleepiness scale; which respectively evaluate OSA and EDS symptoms, were administered to individuals hospitalized at an acute psychiatric treatment unit at the AUB-MC between the dates of January 2014 and October 2016. Additional data collected included general demographics, psychiatric diagnoses, and questionnaires evaluating depression and anxiety symptoms. Statistical analyses utilizing SPSS were performed to determine the prevalence of OSA and EDS, as well as their respective associations with patient profiles.

Results: Our results showed that $39.5 \%$ of participants were found to have a high risk of sleep apnea and $9.9 \%$ of the participants were found to have abnormal daytime sleepiness. The risk of developing OSA was associated with a higher body mass index (BMI) $(P=0.02)$, and depression severity (patient health questionnaire 9 score) $(P=0.01)$. Increasing severity of depressive symptoms was associated with a higher risk of sleep apnea $(P=0.01)$. BMI (odds ratio $[\mathrm{OR}]=5.97,95 \%$ confidence interval $[\mathrm{CI}] 1.89-18.82)$ and depression severity $(\mathrm{OR}=4.04$, 95\% CI 1.80-9.07) were also found to be predictors of OSA. The psychiatric diagnoses of the participants were not found to have a significant association with the risk of sleep apnea.

Conclusion: The risk of OSA is increased among hospitalized psychiatric patients, and this condition can have detrimental effects on psychiatric patients. OSA appears to be underrecognized in this population, psychiatrists should screen for OSA in hospitalized psychiatric patients and refer them for diagnostic testing or treatment when indicated.

Keywords: obstructive sleep apnea, excessive daytime sleepiness, depression, anxiety, psychiatric patients, inpatient psychiatry

\section{Introduction}

Recent findings indicate a potential bi-directional relationship between psychiatric disorders and sleep disorders. ${ }^{1-3}$ Obstructive sleep apnea (OSA), the most common type of sleep apnea, consists of repetitive episodes of upper airway obstruction occurring during sleep. ${ }^{4}$ Upper airway obstruction in OSA is attributed to excess soft tissue in the oropharynx, pharyngeal muscle dysfunction, or anatomical anomalies. ${ }^{4,5}$ Excessive body weight and an elevated body mass index (BMI) have been found to be major predisposing factors for OSA. ${ }^{6}$ The prevalence of OSA is well documented in the medical literature ranging from $5 \%$ in women to $7 \%$ in men. ${ }^{7}$ There is sparse data
Correspondence: Farid R Talih American University of Beirut, 3 Dag Hammarskjold Plaza, 8th Floor, New York, NY 10017-2303, USA Emailft10@aub.edu.lb 
regarding the prevalence of OSA in our region (Middle East). In a study of Saudi patients with renal disease, the prevalence of OSA was $\sim 20 \%$ compared to 5 to $7 \%$ among the general population. ${ }^{8,9}$ Sleep disturbances is a major diagnostic criterion in many mental health disorders. Research has shown an association between depressive symptoms and OSA. ${ }^{10}$ Depressive symptomatology has been documented in $21.5 \%$ of patients surveyed for sleep apnea. ${ }^{11}$ Additionally, a high prevalence of depressive symptoms was detected among individuals with a high likelihood of OSA. ${ }^{12,13}$ Little information concerning the relationship between psychiatric symptoms and sleep apnea exists in the Middle East region in general, and in Lebanon in particular (where this study was conducted).

Another symptom frequently reported in sleep disorders and psychiatric disorders is excessive daytime sleepiness (EDS). Psychiatric disorders have been found to account for 5 to $7 \%$ of EDS; with a higher prevalence in females. ${ }^{14}$ The prevalence of EDS in depression ranges from 5 to $50 \%$ depending on the definition of hyper-somnolence and how it is measured. ${ }^{1,15}$ EDS is commonly measured using the Epworth sleepiness scale (ESS). ${ }^{16}$ The normal range of daytime sleepiness was found, internationally, to correspond to an ESS score of less than $10 .{ }^{17-19}$ A Middle Eastern study reported EDS prevalence at $20 \%$, with a higher prevalence among women. ${ }^{20}$

Most data regarding sleep disorders in psychiatric patients are usually collected from the general (outpatient) psychiatric population; among those with severe mental illness (hospitalized), little is known about the prevalence of sleep disorders. Individuals with severe mental illness are a high-risk group for poor health in general, with reduced access to preventative medical care for multiple reasons, including stigma and low socioeconomic status. Sleep-related complaints in this high-risk population are frequently dismissed as normal or as unavoidable consequences of psychotropic medications, lifestyle, or the mental illness itself. Identifying and treating sleep disorders in hospitalized psychiatric patients may improve outcomes, overall health, and quality of life in a vulnerable population.

The objective of this study was to portray a high prevalence of OSA and EDS among hospitalized psychiatric patients, higher than that of the general population as well as the general, non-hospitalized, psychiatric population. We attempted to investigate the risk of OSA, the prevalence of EDS, and associated variables in this population.

\section{Methods}

The participants in this study were patients hospitalized at the acute psychiatric unit at the American University of Beirut Medical Center (AUB-MC) in Beirut, Lebanon. Following AUB-MC institutional review board approval, participant recruitment began. Over a period of 2 years all eligible patients admitted to the psychiatric unit were asked to participate. The research project, its risks, and benefits were explained to the participants before obtaining written informed consent from all of the participants. The study was a cross-sectional survey consisting of self-reported questionnaires and was administered by trained research assistants. Exclusion criteria included: being younger than 18 years of age, having a dementia diagnosis, and intellectual impairment that would prevent the individual from participating meaningfully in this study. Demographic and general medical information were included in the questionnaire; as well as alcohol and substance use including: caffeine, nicotine, and illicit drug use. The native language of the participants is Arabic and the questionnaires used in this study have been validated in Arabic speakers and Arab populations.

Psychiatric diagnoses upon admission to the psychiatric unit were also collected. The Berlin questionnaire was used to assess the risk of sleep apnea, and was used as a surrogate to test for sleep disordered breathing among the participants. ${ }^{21}$ The Berlin questionnaire is divided into three categories, patients with positive scores in at least two of these categories are considered to have a high risk of sleep apnea. The first category addresses the presence and intensity of snoring, the second category addresses sleep-related fatigue, and the third category addresses the presence or absence of elevated blood pressure. ${ }^{21}$ The Berlin questionnaire has been validated in Arabic speaking populations. ${ }^{22}$

The ESS was used to evaluate EDS. ${ }^{16}$ It consists of eight items describing different situations in which the individual would have a likelihood of dozing off. Each item is scored from 0 to 3 , with 3 being the highest chance of dozing off. A score greater than 10 suggests abnormal daytime sleepiness. ${ }^{16}$ The ESS has been validated in Arabic speakers. $^{23}$

The patient health questionnaire 9 (PHQ-9) was used to evaluate depressive symptoms. ${ }^{24}$ It includes nine items; for each item participants indicate if the described symptom had bothered them "not at all", "several days", "more than half the days", or "nearly every day" over the past 2 weeks. Scores per item range from 0 to 3 . Scores in the following 
ranges: 10 to $14 ; 15$ to 19 ; and 20 or more correspond to moderate, moderately severe, and severe depressive symptoms, respectively. The PHQ-9 has a sensitivity of $93 \%$ and a specificity of $88 \%$ when used to evaluate depressive symptomatology. ${ }^{24}$ The generalized anxiety disorder 7 (GAD-7) survey, ${ }^{25}$ a 7-item scale, is a screening tool for anxiety. ${ }^{25}$ For each item, participants indicate whether, during the past 2 weeks, an anxiety symptom bothered them: "not at all", "several days", "more than half the days", or "nearly every day". Scores of 5, 10, and 15 are taken as the cutoff points for mild, moderate, and severe anxiety. The GAD-7 has a sensitivity of $89 \%$ and a specificity of $82 \%$ for detecting anxiety symptoms. ${ }^{25}$ The GAD-7 and PHQ-9 have both been validated in Arabic speaking populations. ${ }^{26}$

Collected data were recorded in an electronic database and analyzed using SPSS ${ }^{\circledR}$ (IBM Corporation, Armonk, NY, USA) for Windows ${ }^{\circledR}$ version 20. The prevalence of OSA, as well as the descriptive analyses for all variables were presented as number and percent. Associations between potential factors and patient characteristics relevant to OSA were assessed using the Pearson Chi square test. Multivariate analyses were performed to determine possible predictors of OSA and EDS. All tests were 2-sided with a type one error of 0.05 .

\section{Results}

The investigators recruited 152 participants from the patients hospitalized at the AUB-MC psychiatric unit. Our psychiatric unit admits $\sim 250$ patients per year. The sample used in this study was collected over 2 years. Some of the patients admitted to the unit did not agree to participate, and some did not meet criteria for inclusion. We estimate a response rate of $\sim 30 \%$. The participants were $56.6 \%$ female and the average age range was 26 to 45 . The demographic and descriptive characteristics of the group are shown in Table 1. As expected, a lower risk of sleep apnea, per the Berlin questionnaire scores, was detected in females when compared to males (Table 1). Most of the participants were overweight, and this group had the highest risk of sleep apnea. The primary psychiatric diagnoses of the participants upon admission to the psychiatric unit are listed in Table 1. Depression was the most common admitting diagnosis at $44.0 \%$ (Table 1). When the participants were asked if they ever received a diagnosis of sleep apnea, or ever used continuous positive airway pressure or bi-level positive airway pressure devices for treatment of OSA, only 5\% responded affirmatively. Per the Berlin questionnaire, $39.5 \%$ of the participants were found to have a high risk of sleep apnea. OSA was found to be associated with BMI $(P=0.02)$ and PHQ-9 scores $(P=0.01)$. As expected, higher BMIs were associated with a higher risk of sleep apnea $(P=0.02)$ (Table 1$)$. Severity of depressive symptoms was also associated with a higher risk of sleep apnea $(P=0.01)$ (Table 1$)$. The psychiatric diagnoses of the participants and anxiety symptoms (per GAD-7) were not found to have a significant association with the risk of sleep apnea (Table 1).

After administering the ESS, only $9.9 \%$ of the participants were found to have abnormal daytime sleepiness (Table 2). Out of 152 patients, $77.6 \%$ slept for less than 7 to 8 hours at night and out of those patients, $46.7 \%$ had an abnormal range of daytime sleepiness (Table 2). Multivariate analyses were done which showed an age range of 18-25 years old, male sex, BMI, and depressive symptoms to be significant predictors of OSA (Table 3 ). The only significant predictor of EDS in this sample was found to be the age range 18-25 years old (Table 4 ).

\section{Discussion}

To the best of our knowledge, this study is the first to assess OSA and EDS among psychiatric patients in the Middle East. The results of this study show a high risk of OSA among hospitalized psychiatric patients. The risk of sleep apnea found in this study (39.5\%) was higher than the prevalence rates of OSA among the general population. Prevalence rates generally range from $5 \%$ in women to $7 \%$ in men, ${ }^{7}$ with prevalence increasing in women as they age. ${ }^{27}$ The prevalence of sleep apnea in this sample was found to be higher in males, which is consistent with the literature (Table 1). The prevalence of sleep apnea was not found to be higher in older patients, which is unusual per the literature. Being in the younger age range was found to be a predictor of OSA (Table 3). Despite having a low odds ratio (OR), this finding was still significant. This underscores the importance of not overlooking OSA symptoms and their risks in the young psychiatric patient population. This could also be explained by the small sample size when compared to larger general population studies. The prevalence of OSA in this study, was found to be higher than available prevalence data from our region regarding OSA which showed a prevalence ranging up to $21 \%$ in special populations, such as end-stage renal failure patients. ${ }^{8,9,28}$ The high prevalence in this study, may be due to factors related to the severity of psychiatric disorders in our sample that can exacerbate sleep apnea; such as the effects of sedating psychotropic medications on breathing during 
Table I Patients' characteristics and risk of sleep apnea per the Berlin questionnaire

\begin{tabular}{|c|c|c|c|}
\hline & $\begin{array}{l}\text { Patients with high risk } \\
\text { of sleep apnea, } n(\%) \\
\mathbf{N}=60\end{array}$ & $\begin{array}{l}\text { Patients with low risk } \\
\text { of sleep apnea, } \mathrm{n}(\%) \\
\mathrm{N}=92\end{array}$ & $P$-value \\
\hline Sex & & & 0.16 \\
\hline Male & $30(50.0)$ & $35(38.5)$ & \\
\hline Female & $30(50.0)$ & $56(61.5)$ & \\
\hline Age (years) & & & 0.06 \\
\hline $18-25$ & $9(15.0)$ & $33(35.9)$ & \\
\hline $26-35$ & $18(30.0)$ & $25(27.2)$ & \\
\hline $36-45$ & $18(30.0)$ & $18(19.6)$ & \\
\hline $46-55$ & $8(13.3)$ & $10(10.9)$ & \\
\hline$>55$ & $7(11.7)$ & $6(6.5)$ & \\
\hline BMI categories & & & 0.02 \\
\hline Underweight & $2(3.3)$ & $8(8.9)$ & \\
\hline Normal & $16(26.7)$ & $40(44.4)$ & \\
\hline Overweight & $27(45.0)$ & $37(4 I .1)$ & \\
\hline Obese & $15(25.0)$ & $5(5.6)$ & \\
\hline Diagnosis & & & 0.33 \\
\hline Depression & $31(5 I .7)$ & $36(39.1)$ & \\
\hline Schizophrenia/schizoaffective disorder & $10(16.7)$ & II (I2.0) & \\
\hline Bipolar & $5(8.3)$ & $19(20.7)$ & \\
\hline Anxiety disorder & $6(10.0)$ & $10(9.8)$ & \\
\hline SUD & $8(13.3)$ & $15(16.3)$ & \\
\hline Eating disorder & $0(0.0)$ & $\mathrm{I}(\mathrm{I} . \mathrm{I})$ & \\
\hline Depressive symptoms & & & 0.01 \\
\hline None & $5(8.3)$ & $18(19.6)$ & \\
\hline Mild to moderate & $10(16.7)$ & $26(28.3)$ & \\
\hline Severe & $45(75.0)$ & $48(52.2)$ & \\
\hline Anxiety symptoms & & & 0.47 \\
\hline No anxiety & $10(35.7)$ & $18(19.6)$ & \\
\hline Mild & $19(31.7)$ & $25(27.2)$ & \\
\hline Moderate & $5(37.5)$ & $15(16.3)$ & \\
\hline Severe & $26(43.3)$ & $34(37.0)$ & \\
\hline Tobacco & $42(70.0)$ & $54(58.7)$ & 0.16 \\
\hline Caffeine & $49(81.7)$ & 77 (83.7) & 0.74 \\
\hline Alcohol & $19(31.7)$ & 31 (33.7) & 0.79 \\
\hline Substance abuse & & & 0.37 \\
\hline Cannabis & $6(10.0)$ & $17(18.5)$ & \\
\hline Cocaine & $\mathrm{I}(\mathrm{I} .7)$ & $0(0.0)$ & \\
\hline Non-prescribed opiates & $\mathrm{I}(\mathrm{I} .7)$ & $\mathrm{I}(\mathrm{I} . \mathrm{I})$ & \\
\hline Non-prescribed benzodiazepines & $4(6.7)$ & $3(3.3)$ & \\
\hline
\end{tabular}

Abbreviations: BMI, body mass index; SUD, substance use disorder.

sleep, and the increased prevalence of metabolic syndrome, obesity, drug and alcohol use in this population. ${ }^{29}$

Obesity is a major predictive factor for sleep apnea, ${ }^{6,8}$ the results of our study are consistent with the literature, with $25.0 \%$ of OSA patients in our sample being obese, and $45.0 \%$ overweight (Table 1). Psychiatric disorders have been shown to contribute to the development and maintenance of sleep apnea. ${ }^{29,30}$ Sleep apnea is associated with fatigue and negatively affects quality of life. ${ }^{31,32}$ Concomitant depression or anxiety worsens the quality of life in patients with sleep apnea, and patients frequently end up in a vicious cycle of worsening sleep and psychiatric symptoms. ${ }^{3}$ The prevalence of OSA in the depressed population is known to be higher than the general population; ${ }^{33}$ the results of our study show that $75.0 \%$ of patients with a high risk of sleep apnea have severe depressive symptoms (Table 1). Sedating psychiatric medications and specifically benzodiazepines, might be a major contributor to the co-existence of sleep apnea in more depressed patients. Benzodiazepines decrease the respiratory effort and directly exacerbate OSA. ${ }^{34}$

Even though the role of serotonin in depression is well established, its contribution to sleep apnea is still questionable. ${ }^{35}$ The bi-directional relationship between depression and OSA may have underlying biological links. 
Table 2 Patients' characteristics and range of daytime sleepiness as per Epworth sleepiness scale

\begin{tabular}{|c|c|c|c|}
\hline & $\begin{array}{l}\text { Patients with normal } \\
\text { range of sleepiness, } n(\%) \\
N=137\end{array}$ & $\begin{array}{l}\text { Patients with abnormal } \\
\text { range of sleepiness, } n(\%) \\
N=15\end{array}$ & $P$-value \\
\hline Sex & & & 0.18 \\
\hline Male & $61(44.9)$ & $4(26.7)$ & \\
\hline Female & $75(55.1)$ & II (73.3) & \\
\hline Age (years) & & & 0.29 \\
\hline $18-25$ & $35(25.5)$ & $7(46.7)$ & \\
\hline $26-35$ & $41(29.9)$ & $2(13.3)$ & \\
\hline $36-45$ & $32(23.4)$ & $4(26.7)$ & \\
\hline $46-55$ & $16(11.7)$ & $2(13.3)$ & \\
\hline$>55$ & $13(9.5)$ & $0(0.0)$ & \\
\hline BMI categories & & & 0.77 \\
\hline Underweight & $9(6.7)$ & I (6.7) & \\
\hline Normal & $52(38.5)$ & $4(26.7)$ & \\
\hline Overweight & $57(42.2)$ & $7(46.7)$ & \\
\hline Obese & $17(12.6)$ & $3(20.0)$ & \\
\hline Sleep hours & & & 0.91 \\
\hline Less than 7 to 8 hours & $69(50.4)$ & $7(46.7)$ & \\
\hline More than 8 hours & $68(49.6)$ & $8(54.3)$ & \\
\hline Diagnosis & & & 0.90 \\
\hline Depression & $62(45.3)$ & $5(33.3)$ & \\
\hline Schizophrenia/schizoaffective disorder & $19(13.9)$ & $2(13.3)$ & \\
\hline Bipolar & $20(14.6)$ & $4(26.7)$ & \\
\hline Anxiety disorder & $13(9.5)$ & $2(13.3)$ & \\
\hline Substance use disorder & $21(15.3)$ & $2(13.3)$ & \\
\hline Eating disorder & $\mathrm{I}(0.7)$ & $0(0.0)$ & \\
\hline Depressive symptoms & & & 0.53 \\
\hline None & $22(95.7)$ & $\mathrm{I}(4.3)$ & \\
\hline Mild to moderate & $33(91.7)$ & $3(8.3)$ & \\
\hline Severe & $82(88.2)$ & $\mathrm{II}(\mathrm{II} .8)$ & \\
\hline Anxiety symptoms & & & 0.84 \\
\hline No anxiety & $26(92.9)$ & $2(7.1)$ & \\
\hline Mild & $40(90.9)$ & $4(9.1)$ & \\
\hline Moderate & $17(85.0)$ & $3(15.0)$ & \\
\hline Severe & $54(90.0)$ & $6(10.0)$ & \\
\hline Tobacco & $86(62.8)$ & $10(66.7)$ & 0.76 \\
\hline Caffeine & $113(82.5)$ & $13(86.7)$ & 0.68 \\
\hline Alcohol & $45(32.8)$ & $5(33.3)$ & 0.97 \\
\hline Substance abuse & & & 0.84 \\
\hline Cannabis & $22(16.1)$ & $\mathrm{I}(6.7)$ & \\
\hline Cocaine & $\mathrm{I}(0.7)$ & $0(0.0)$ & \\
\hline Non-prescribed opiates & $2(1.5)$ & $0(0.0)$ & \\
\hline Non-prescribed benzodiazepines & $6(4.4)$ & $\mathrm{I}(6.7)$ & \\
\hline
\end{tabular}

Abbreviation: BMI, body mass index.

Table 3 Multivariate analysis of potential predictors of sleep apnea

\begin{tabular}{lll}
\hline & \multicolumn{2}{l}{$\begin{array}{l}\text { Obstructive sleep apnea } \\
\text { (reference: low) }\end{array}$} \\
\cline { 2 - 3 } & OR $(95 \%$ CI) & P-value \\
\hline Age I8-25 years & $0.29(0.12-0.7 I)$ & 0.01 \\
Sex & $2.06(0.98-4.34)$ & 0.06 \\
BMI - obese & $5.97(1.89-18.82)$ & 0.002 \\
Depressive symptoms & $4.04(1.80-9.07)$ & 0.001 \\
\hline
\end{tabular}

Notes: Variables included in the model were: age (reference: 26-35 years), sex (reference: female); BMI (reference: normal); depressive symptoms (reference: none); anxiety (reference: none); tobacco (reference: none); caffeine (reference: none); alcohol (reference: none).

Abbreviations: $\mathrm{BMI}$, body mass index; $\mathrm{OR}$, odds ratio; $\mathrm{Cl}$, confidence interval.
Inflammatory markers such as TNF-alpha, C-reactive protein (CRP), and IL-6 have been shown to be elevated in OSA patients and similarly in depression, and they may link depression and OSA. ${ }^{36,37}$ Specific inflammatory cytokines have also been linked to obesity, depression, and OSA. ${ }^{38}$ The persistent elevation of these inflammatory markers in individuals who have both disorders might perpetuate refractory psychiatric symptoms.

The prevalence of EDS in this sample was approximately $10 \%$, which is consistent with reported hypersomnia in 
Table 4 Multivariate analysis of potential predictors of excessive daytime sleepiness

\begin{tabular}{|c|c|c|}
\hline & \multicolumn{2}{|c|}{$\begin{array}{l}\text { Range of sleepiness } \\
\text { (reference: normal) }\end{array}$} \\
\hline & OR (95\% CI) & P-value \\
\hline Age $18-25$ years & $2.55(0.86-7.54)$ & 0.09 \\
\hline \multicolumn{3}{|c|}{$\begin{array}{l}\text { Notes: Variables included in the model were: age (reference: } 26-35 \text { years), sex } \\
\text { (reference: female); BMI (reference: normal); depressive symptoms (reference: } \\
\text { none); anxiety (reference: none); tobacco (reference: none); caffeine (reference: } \\
\text { none); alcohol (reference: none). }\end{array}$} \\
\hline
\end{tabular}

psychiatric disorders; albeit in the lower range. ${ }^{1,15}$ Abnormal daytime sleepiness in this sample was not found to be associated with any of the tested variables (Table 2). This was an unusual finding considering that EDS has been shown to be associated with some psychiatric disorders and psychotropic medications. ${ }^{14,39}$ This finding could be attributed to underreporting by our participants of their EDS. Despite most patients in this study sleeping less than 7 to 8 hours per day, there was no significant association between reported sleep time and EDS. A possible explanation for this finding may be habituation to chronic EDS in this patient population. Objective measures of EDS, such as polysomnography with mean sleep latency testing, would be able to further quantify daytime sleepiness in this patient population. The relatively lower prevalence of EDS could also potentially be attributed to positive behavioral changes, and improved sleep hygiene that the participants are exposed to during their stay at the psychiatric unit. Switching or adjusting the patients' psychotropic medications during hospitalization might also have contributed to the lower prevalence of EDS in this sample. In a previous study, patients with substance abuse were found to be at a very high risk of experiencing sleep disorders. ${ }^{40}$ Even though EDS in this sample was not found to be associated with drug abuse, the percentage of patients who reported illicit drug use in this sample was low. Overall, EDS in psychiatric patients is very challenging to evaluate and treat. In some cases of severe hypersomnia, the culprit was found to be narcolepsy, and due to overlap in symptoms some patients were misdiagnosed as schizophrenics, and the treatment of the narcolepsy-related hypersomnia was overlooked or delayed. ${ }^{41}$

OSA was found to be associated with depressive symptomatology in general, but there was no significant association with specific psychiatric diagnoses in our sample. This could be attributed to the relatively small sample size. Another limitation to this study, is not taking into account the specific types of psychotropic medications being administered to the patients and their potential effects on sleep. Data on prescribed medications were collected; however, to be able to obtain statistically meaningful results from analyzing the effects of medications in our population, an unfeasibly large sample size would be required given the heterogeneity of medications prescribed for the multiple psychiatric disorders. This was a questionnaire-based study, which by itself is a significant limitation, incorporating objective measures such as actigraphy and polysomnography in a future study will provide more robust data. This study may have under-reported sleep disordered breathing in general, by not detecting other types of apnea, mainly central sleep apnea. Central sleep apnea may be quite prevalent in this population since many participants are prescribed centrally acting depressants.

Sleep disorders in general and sleep disordered breathing in particular among psychiatric patients, are frequently overlooked or underestimated. In a recent study by our group, restless legs syndrome was found to be highly prevalent among hospitalized psychiatric patients at $18 \%$, and was associated with suicidal ideation. ${ }^{42}$

\section{Conclusion}

Among the patients who were found to have a high risk of sleep apnea in this study, only a minimal percentage of them received a previous diagnosis of sleep apnea or were currently being treated for sleep apnea; this finding underscores the need to increase screening for sleep disorders in this vulnerable population. It is also important to address OSA in this population; since OSA is associated with increased cardiovascular morbidity, and will increase morbidity in psychiatric patients who are already at a higher risk of increased metabolic and cardiovascular diseases. The biological factors underlying the relationship between sleep and psychiatric disorders are multifactorial and complex, however it is important to improve screening and offer appropriate therapeutic intervention to improve outcomes and quality of life in psychiatric patients.

\section{Acknowledgment}

The authors are thankful for the staff of the inpatient psychiatry unit at AUB-MC for facilitating this work and assisting the investigators in data collection.

\section{Author contributions}

FRT, JJA, and FHK worked on study conception and design. FRT, JJA, and HMT analyzed and interpreted the data. FRT, JJA, HMT, and FHK critically revised the manuscript with input from the entire team. All authors have read and approved the final draft. 


\section{Disclosure}

The authors report no conflicts of interest in this work.

\section{References}

1. Liu X, Buysse DJ, Gentzler AL, et al. Insomnia and hypersomnia associated with depressive phenomenology and comorbidity in childhood depression. Sleep. 2007;30(1):83-90.

2. Batterham PJ, Glozier N, Christensen H. Sleep disturbance, personality and the onset of depression and anxiety: prospective cohort study. Aus N Z J Psychiatry. 2012;46(11):1089-1098.

3. Akashiba T, Kawahara S, Akahoshi T, et al. Relationship between quality of life and mood or depression in patients with severe obstructive sleep apnea syndrome. Chest. 2002;122(3):861-865.

4. Berry RB, Brooks R, Gamaldo CE, Harding SM, Marcus C, Vaughn B. The AASM manual for the scoring of sleep and associated events. Rules, Terminology and Technical Specifications, Darien, Illinois, American Academy of Sleep Medicine. 2012. Available from: http://www.aasmnet org/scoringmanual/default.aspx. Accessed October 1, 2016.

5. Berry RB. Dreaming about an open upper airway. Sleep. 2006;29(4): 429-431.

6. Young T, Peppard PE, Taheri S. Excess weight and sleep-disordered breathing. J Appl Physiol (1985). 2005;99(4):1592-1599.

7. Young T, Palta M, Dempsey J, Skatrud J, Weber S, Badr S. The occurrence of sleep-disordered breathing among middle-aged adults. N Engl J Med. 1993;328(17):1230-1235.

8. Al-Jahdali H. Prevalence of sleep apnea and excessive day time sleepiness in patients with end-stage renal disease on dialysis. Saudi J Kidney Dis Transpl. 2012;23(2):251-261.

9. Mahboub B, Afzal S, Alhariri H, Alzaabi A, Vats M, Soans A. Prevalence of symptoms and risk of sleep apnea in Dubai, UAE. Int J Gen Med. 2013;6:109-114.

10. Pan ML, Tsao HM, Hsu CC, et al. Bidirectional association between obstructive sleep apnea and depression: A population-based longitudinal study. Medicine (Baltimore). 2016;95(37):e4833.

11. Acker J, Richter K, Piehl A, Herold J, Ficker JH, Niklewski G. Obstructive sleep apnea (OSA) and clinical depression-prevalence in a sleep center. Sleep Breath. Epub 2016 Oct 5.

12. Farajzadeh M, Hosseini M, Mohtashami J, Chaibakhsh S, Zagheri Tafreshi M, Ghanei Gheshlagh R. The association between obstructive sleep apnea and depression in older adults. Nurs Midwifery Stud. 2016;5(2):e32585.

13. BaHammam AS, Kendzerska T, Gupta R, et al. Comorbid depression in obstructive sleep apnea: an under-recognized association. Sleep Breath. 2016;20(2):447-456.

14. Kaplan KA, Harvey AG. Hypersomnia across mood disorders: a review and synthesis. Sleep Med Rev. 2009;13(4):275-285.

15. Kotagal S. Hypersomnia in children: interface with psychiatric disorders. Child Adolesc Psychiatr Clin N Am. 2009;18(4):967-977.

16. Johns MW. A new method for measuring daytime sleepiness: the Epworth sleepiness scale. Sleep. 1991;14(6):540-545.

17. Chen SY, Clift SJ, Dahlitz MJ, Dunn G, Parkes JD. Treatment in the narcoleptic syndrome: self assessment of the action of dexamphetamine and clomipramine. J Sleep Res. 1995;4(2):113-118.

18. Manni R, Politini L, Ratti MT, Tartara A. Sleepiness in obstructive sleep apnea syndrome and simple snoring evaluated by the Epworth Sleepiness Scale. J Sleep Res. 1999;8(4):319-320.

19. Izci B, Ardic S, Firat H, Sahin A, Altinors M, Karacan I. Reliability and validity studies of the Turkish version of the Epworth Sleepiness Scale. Sleep Breath. 2008;12(2):161-168.

20. Fatani A, Al-Rouqi K, Al Towairky J, et al. Effect of age and gender in the prevalence of excessive daytime sleepiness among a sample of the Saudi population. J Epidemiol Glob Health. 2015;5(4 Suppl 1): S59-S66.
21. Netzer NC, Stoohs RA, Netzer CM, Clark K, Strohl KP. Using the Berlin Questionnaire to identify patients at risk for the sleep apnea syndrome. Ann Intern Med. 1999;131(7):485-491.

22. Saleh AB, Ahmad MA, Awadalla NJ. Development of Arabic version of Berlin questionnaire to identify obstructive sleep apnea at risk patients. Ann Thorac Med. 2011;6(4):212-216.

23. Al-Abri M, Al-Hamhami A, Al-Nabhani H, Al-Zakwani I. Validation of the arabic version of the epworth sleepiness scale in oman. Oman Med J. 2013;28(6):454-456.

24. Spitzer RL, Kroenke K, Williams JB. Validation and utility of a selfreport version of PRIME-MD: the PHQ primary care study. Primary Care Evaluation of Mental Disorders. Patient Health Questionnaire. JAMA. 1999;282(18):1737-1744.

25. Spitzer RL, Kroenke K, Williams JB, Löwe B. A brief measure for assessing generalized anxiety disorder: the GAD-7. Arch Intern Med. 2006;166(10):1092-1097.

26. Sawaya H, Atoui M, Hamadeh A, Zeinoun P, Nahas Z. Adaptation and initial validation of the Patient Health Questionnaire - 9 (PHQ-9) and the Generalized Anxiety Disorder - 7 Questionnaire (GAD-7) in an Arabic speaking Lebanese psychiatric outpatient sample. Psychiatry Res. 2016;239:245-252.

27. Punjabi NM. The epidemiology of adult obstructive sleep apnea. Proc Am Thorac Soc. 2008;5(2):136-143.

28. BaHammam AS, Alrajeh MS, Al-Jahdali HH, BinSaeed AA. Prevalence of symptoms and risk of sleep apnea in middle-aged Saudi males in primary care. Saudi Med J. 2008;29(3):423-426.

29. Atkeson AD, Basner RC. Sleep and quality of life in sleep apnea. Sleep and Quality of Life in Clinical Medicine. 2008:79-91.

30. Yosunkaya S, Kutlu R, Cihan FG. Evaluation of depression and quality of life in patients with obstructive sleep apnea syndrome. Niger J Clin Pract. 2016;19(5):573-579.

31. Braley TJ, Segal BM, Chervin RD. Obstructive sleep apnea and fatigue in patients with multiple sclerosis. J Clin Sleep Med. 2014;10(2):155.

32. Stelmach-Mardas M, Mardas M, Iqbal K, Tower RJ, Boeing H, Piorunek T. Quality of life, depression and dietary intake in obstructive sleep apnea patients. Health Qual Life Outcomes. 2016;14(1):111.

33. Gupta MA, Simpson FC. Obstructive sleep apnea and psychiatric disorders: a systematic review. J Clin Sleep Med. 2015;11(2):165-175.

34. Berry RB, Kouchi K, Bower J, Prosise G, Light RW. Triazolam in patients with obstructive sleep apnea. Am J Respir Crit Care Med. 1995; 151(2 Pt 1):450-454.

35. Schröder CM, O’Hara R. Depression and obstructive sleep apnea (OSA). Ann Gen Psychiatry. 2005;4:13.

36. Vgontzas AN, Zoumakis E, Bixler EO, et al. Adverse effects of modest sleep restriction on sleepiness, performance, and inflammatory cytokines. J Clin Endocrinol Metab. 2004;89(5):2119-2126.

37. Patel SR, Zhu X, Storfer-Isser A, et al. Sleep duration and biomarkers of inflammation. Sleep. 2009;32(2):200-204.

38. Soczynska JK, Kennedy SH, Woldeyohannes HO, et al. Mood disorders and obesity: understanding inflammation as a pathophysiological nexus. Neuromolecular Med. 2011;13(2):93-116.

39. Schweitzer P. Drugs that disturb sleep and wakefulness. In: Kryger MH, Roth T, Dement WC. Principles and Practice of Sleep Medicine. Elsevier; 2000:542-560.

40. Mahfoud Y, Talih F, Streem D, Budur K. Sleep disorders in substance abusers: how common are they? Psychiatry (Edgmont). 2009; 6(9):38-42.

41. Talih FR. Narcolepsy presenting as schizophrenia: a literature review and two case reports. Innov Clin Neurosci. 2011;8(4):30-34.

42. Talih F, Ajaltouni J, Kobeissy F. Restless leg syndrome in hospitalized psychiatric patients in Lebanon: a pilot study. Neuropsychiatr Dis Treat. 2016;12:2581 


\section{Publish your work in this journal}

Neuropsychiatric Disease and Treatment is an international, peerreviewed journal of clinical therapeutics and pharmacology focusing on concise rapid reporting of clinical or pre-clinical studies on a range of neuropsychiatric and neurological disorders. This journal is indexed on PubMed Central, the 'PsycINFO' database and CAS, and is the official journal of The International Neuropsychiatric Association (INA). The manuscript management system is completely online and includes a very quick and fair peer-review system, which is all easy to use. Visit http://www.dovepress.com/testimonials.php to read real quotes from published authors.

\footnotetext{
Submit your manuscript here: http://www.dovepress.com/neuropsychiatric-disease-and-treatment-journal
} 